\section{Design of FRET-TaqMan probes for multiplex real-time PCR using an internal positive control}

Prithiviraj Jothikumar ${ }^{1}$, Vincent Hill ${ }^{2}$, and Jothikumar Narayanan ${ }^{2}$ ${ }^{1}$ Georgia Institute of Technology, Department of Biomedical Engineering, Atlanta, GA, USA and ${ }^{2}$ Centers for Disease Control and Prevention, National Center for Zoonotic, Vector-borne, and Enteric Diseases, Division of Parasitic Diseases, Atlanta, GA, USA

BioTechniques 46:519-524 (June 2009) doi 10.2144/000113127

Keywords: real-time PCR; hybridization probes; FRET; TaqMan; fluorescence; internal control; inhibitors

The multiplexing capabilities with different fluorescent dyes are limited in real-time PCR instruments equipped with one excitation source. Considering this limitation, a design was developed to create a triple-labeled probe as an internal positive control (IPC) that utilizes a combination of the fluorescence resonance energy transfer (FRET) and TaqMan techniques. The IPC probe, labeled with FAM and Cy5.5 fluorophores at the $5^{\prime}$ end and Black Hole Quencher (BHQ) at the $3^{\prime}$ end, enabled Cy5.5 emission through energy transfer from the FAM fluorophore. The second, target-specific TaqMan assay in the multiplex used a FAM- and BHQ1labeled probe at the $5^{\prime}$ and $3^{\prime}$ ends, respectively. Thus, one excitation source was used to generate two different fluorescence emissions (FAM and Cy5.5) that were measured in two separate channels by the real-time PCR instrument. This method can facilitate the development of a low-cost portable handheld real-time PCR instrument capable of multiplex real-time PCR assays using a single excitation source.

\section{Introduction}

Real-time PCR enables continuous monitoring of fluorophore fluorescence during the generation of PCR products in a closed tube format. Currently, available methods utilize either labeled probes or DNA intercalating dye to monitor the amplification of PCR product. The TaqMan probe is a single-stranded oligonucleotide containing a fluorophore and quencher placed 10-30 bases apart. The TaqMan probe is hydrolyzed during the amplification due to the $5^{\prime}-3^{\prime}$ double-strand-specific exonuclease activity of the Taq polymerase that separates the fluorophore from the quencher, resulting in fluorescence increase. Real-time PCR instruments are equipped with fluorescence detectors and software capable of estimating the cycle threshold $(\mathrm{Ct})$, the cycle at which fluorescence is greater than background fluorescence, for positive reactions. However, the technique cannot differentiate a true negative result from a false negative when PCR is affected by amplification inhibitors. After nucleic acid extraction, it is reported that inhibitors may still be present from clinical samples (e.g., hemoglobin), environmental samples (e.g., humic and fulvic acids), and chemicals employed during nucleic acid extraction (e.g., ethanol, detergents, or chaotropic agents). The reliability of diagnostic assays is increased by the inclusion of an internal control nucleic acid that can indicate the presence and impact of PCR inhibitors $(1,2)$. An internal positive control (IPC) is amplified simultaneously in the presence of a target sequence using a labeled fluorophore that emits light at a different wavelength than the fluorophore used for the target sequence assay, with the two fluorophores detected in different channels by the real-time PCR instrument. The commonly used internal control for PCR is a plasmid that contains a sequence similar to that of the assay target except for probe region. A limited number of IPC molecules are added to individual assay target and co-amplified with the target nucleic acid; thus, a positive IPC signal is evidence that the amplification reaction proceeded sufficiently to generate a positive signal from very small quantities of target nucleic acid. This feature is important to ensure equivalent amplification of the IPC and the target nucleic acid (3-9). However, some devices - such as the LightCycler 1.2 and LightCycler 2 (Roche Applied Science, Indianapolis, IN, USA), the Ruggedized Advanced Pathogen Identification Device (R.A.P.I.D.) instrument (Idaho Technology, Salt Lake City, UT, USA), and handheld real-time PCR instruments - are only equipped with one light source and associated emission channel. Multiplexing on these types of instruments requires the use of multi-labeled oligonucleotides and the fluorescence resonance energy transfer (FRET) concept. However, FRET probes can be challenging to design and may lack robustness in hypervariable regions and when non-optimal amplification conditions are present. The use of FRET probes requires the identification of two regions for two probes. The FRET probe system relies on adjacent hybridization of oligonucleotides of the two singlelabeled probes in such a way the fluorescence of the acceptor dye is detected through the excitation of the fluorophore attached to the second probe. A major disadvantage of the FRET probe is the requirement of a larger sequence area necessary to accommodate two adjacent probes with a gap of 2-5 nucleotides. The possibility exists for multiplexing with other label systems, such as MegaStokes dye (DYXL; Dyomics, Jena, Germany) and Pulsar 650 (Biosearch Technologies, Inc., Novato, CA, USA), but these labeling systems require spectral information to compensate for channel crosstalk and are not widely available. The FRETTaqMan probe developed in this study, however, does not require color compensation software and uses fluorophores (FAM, Cy5.5) that are widely available. The present report focuses on the design of a triple-labeled probe to enable multiplexing in instruments equipped with only one blue light emitting diode (LED) excitation source $(470 \mathrm{~nm})$ and associated three corresponding detection channels (e.g., $530 \mathrm{~nm}$, $640 \mathrm{~nm}$, and $705 \mathrm{~nm}$ ).

\section{Materials and methods}

\section{Probe design principle}

All experiments were performed on Idaho Technology's R.A.P.I.D. system, a 32-sample portable real-time PCR instrument 

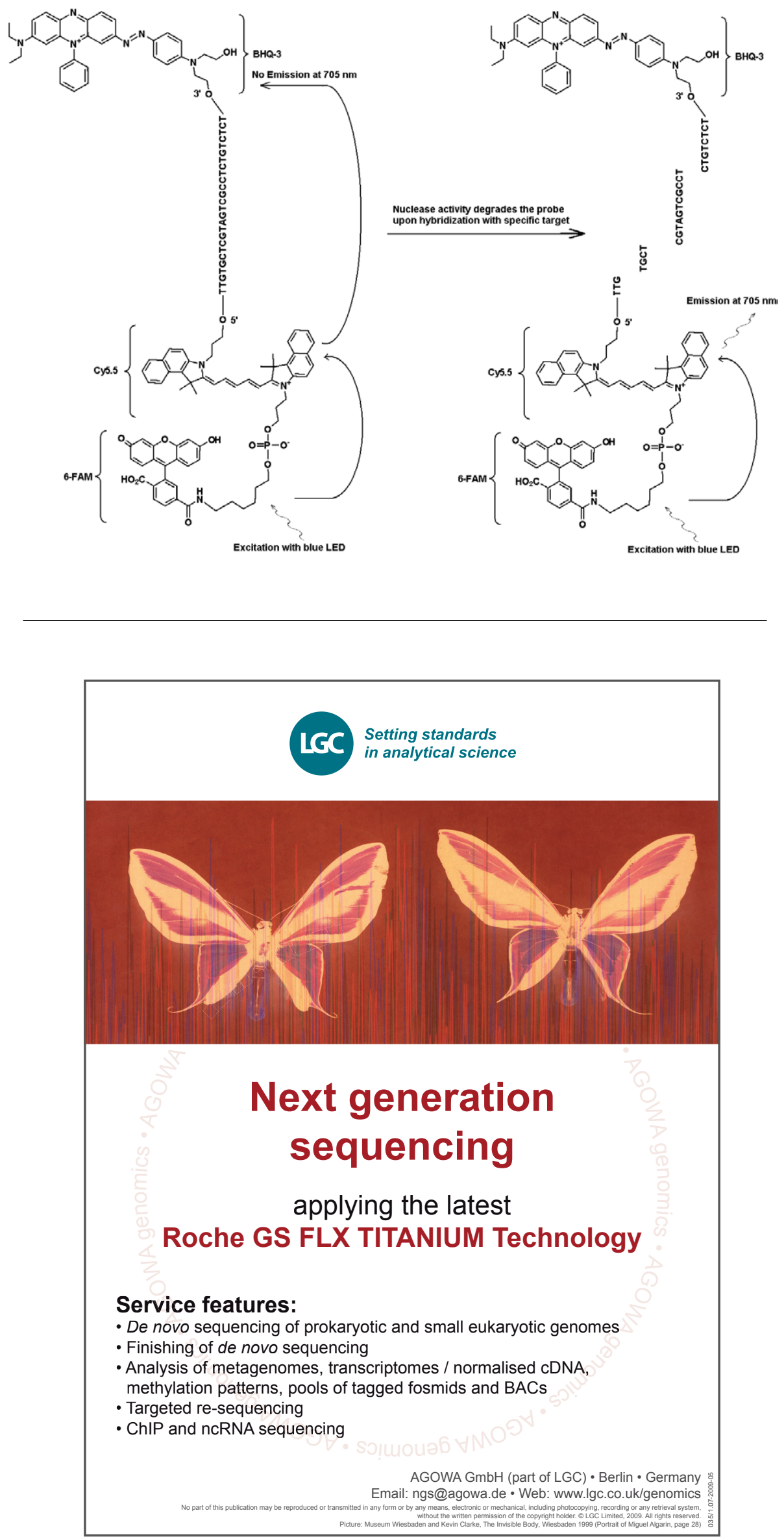

Figure 1. Mechanism of wavelength-shifting FRET-TaqMan probe. The FRET-TaqMan probe contains three labels: a black hole quencher moiety at the end of the $3^{\prime}$ arm, an emitter fluorophore (Cy5.5), and a harvester fluorophore (FAM) joined together at the end of its $5^{\prime}$ arm. The FAM efficiently absorbs energy from blue LED as a light source. In the absence of targets, the probe is dark because the energy absorbed by the FAM is transferred to Cy5.5 and in turn, the energy released from Cy5.5 is transferred to the quencher and is lost as heat energy. In the presence of targets, the TaqMan probe is cleaved by the activity of $T a q$ polymerase and quencher is released and the energy absorbed by the FAM is transferred to Cy5.5 through FRET mechanism to emit fluorescence at $705 \mathrm{~nm}$.

equipped with a blue LED for excitation $(470 \mathrm{~nm})$ and associated three fluorescence detection channels that measure fluorescence at 530, 640, and $705 \mathrm{~nm}$ for monoand dual-color assays. Primers JVAF and JVAR (10) and probe 5'-FAM/BHQ-1 were synthesized at the CDC Biotechnology Core Facility (Atlanta, GA, USA) and probe 5'FAM-Cy5.5/3'BHQ-3 was synthesized at Idaho Technology. To enable duplex TaqMan PCR, one probe was labeled with 5'-FAM/BHQ-1 (520-nm fluorescence wavelength) and another one with 5'FAM-Cy5.5/3'BHQ-3 (705-nm fluorescence wavelength). In solution, the probe labeled with 5'FAM-Cy5.5/3'BHQ-3 remains quenched upon hybridization to a sequence, and upon subsequent cleavage of the probe by enzymatic primer extension, the quencher is eliminated. This results in FRET through the excitation of FAM by the light source of the R.A.P.I.D. instrument. The excitation energy is transferred to the acceptor fluorophore, Cy5.5, and the emitted fluorescence is measured in a second fluorescence channel (fluorescence channel F3 for the R.A.P.I.D. instrument). The emission of Cy5.5 fluorescence is the result of the combination of FRET and TaqMan probe techniques, so these triplelabeled probes can be referred to as "FRETTaqMan" probes.

Primers, probes, and plasmid

To demonstrate the multiplexing capabilities of the FRET-TaqMan design, we used a genus-specific Cryptosporidium real-time PCR assay to amplify a 159-bp portion of the $18 S$ rRNA gene (10). The forward primer (JVAF) was 5'-ATGACGGGTAACGGGGAAT-3', reverse primer (JVAR) was 5'-CCAATTACAAAACCAAAAAGTCC-3', and TaqMan probe (JVAP) was 5'-FAM-CGCGCCTGCTGCCTTCCTTAGATG-BHQ1-3', corresponding to nucleotides 100-118, 258-236, and 184-161, respectively, of 

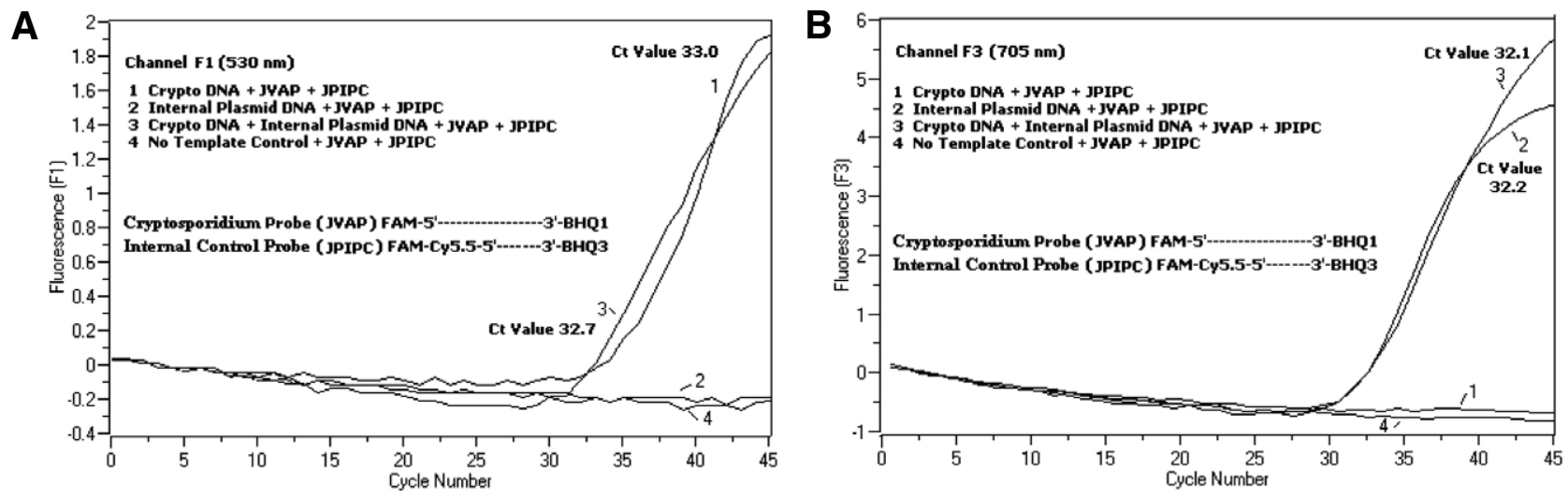

Figure 2. Real-time monitoring profiles using two different probe combinations. An equimolar mixture of a FAM/BHQ1 and a FAM/Cy5.5/BHQ3 was added in all PCR reaction mixtures. (A) Channel F1 monitors increase in FAM fluorescence for the presence of $C$. parvum. Plot 1 (channel F1 primarily collects FAM signal) containing only $C$. parvum DNA revealed Cryptosporidium-specific FAM signal in the PCR. Plot 2 contained only plasmid DNA and no FAM signal from FAM/Cy5.5/BHQ3 probe. Plot 3 contained both $C$. parvum and plasmid DNA but produced only $C$. parvum-specific FAM signal. Plot 4 was a negative sample containing only nuclease-free water in place of any template DNA; no fluorescence was observed from the probes. (B) Channel F3 monitors increase in Cy5.5 for the presence of internal control plasmid DNA. Plot 1 contained only C. parvum DNA and no fluorescence signal from FAM/ BHQ1 probe. Plot 2 (channel F3 primarily collects Cy5.5 signal) containing only plasmid DNA revealed internal plasmid control (IPC)-specific Cy5.5 signal in the PCR. Plot 3 contained both $C$. parvum and plasmid DNA but produced only IPC-specific Cy5.5 signal. Plot 4 was a negative sample containing only nuclease free water in place of any template DNA; no fluorescence was observed from the probes.

GenBank accession no. AY458612. Primers for the construction of the Cryptosporidium IPC contained the same set of primer sequences reported for amplification of Cryptosporidium spp., except for the probebinding region of the target DNA, which was replaced with the artificial sequence 5'-TTGTGCTCGTAGTCGCCTCTGTCTCT-3' (JPIPC). This probe sequence had an annealing temperature of $69^{\circ} \mathrm{C}$ and was designed to have no secondary structure. Theoretical specificity for internal probe was ascertained by a BLAST (11) search of the probe and primer sequences in which no exact match was found with any Cryptosporidium sequence. The IPC fragment was synthesized and cloned into a plasmid by Integrated DNA Technologies (Coralville, IA, USA). A known concentration of plasmid DNA (100 copies/reaction) was added into the master mix of all reactions except in negative control. A triple-labeled probe (JPIPC) specific to the IPC was synthesized by Idaho Technology, as shown in Figure 1. The FAM/Cy5.5/BHQ3 oligonucleotide probe was synthesized starting on the $3^{\prime}$ end with non-fluorescent Black
Hole Quencher (BHQ-3) controlled pore glass (CPG). The $5^{\prime}$ end was then conjugated with a Cy 5.5 phosporamidite followed by a terminal 5 ' fluorescein (6-FAM) phosporamidite.

\section{Real-time PCR assay}

Flow cytometry sorted Cryptosporidium parvum oocysts (200 oocysts per tube) obtained from the University of Wisconsin's State Laboratory of Hygiene (Madison, WI, USA). DNA from C. parvum oocysts was extracted using a protocol described by Hill et al. (12) and suspended in $80 \mu \mathrm{L}$ Tris EDTA (TE, pH 8.0) buffer. Two microliters of DNA were added per reaction, which corresponded to 5 oocysts (equivalent to 100 copies of target DNA). The amplification reaction mixture consisted of Quantifast Probe PCR + ROX kit reaction mixture (Cat. no. 204354; Qiagen) with no ROX added, JVAF, JVAR primers (0.4 $\mu \mathrm{M}$ each), JVAP probe $(0.2 \mu \mathrm{M})$, JPIPC probe $(0.2 \mu \mathrm{M})$, and 100 copies of the IPC. An aliquot $(2 \mu \mathrm{L})$ of the extracted DNA sample was added to the reaction capillary (Roche Diagnostics, Indianapolis, IN,

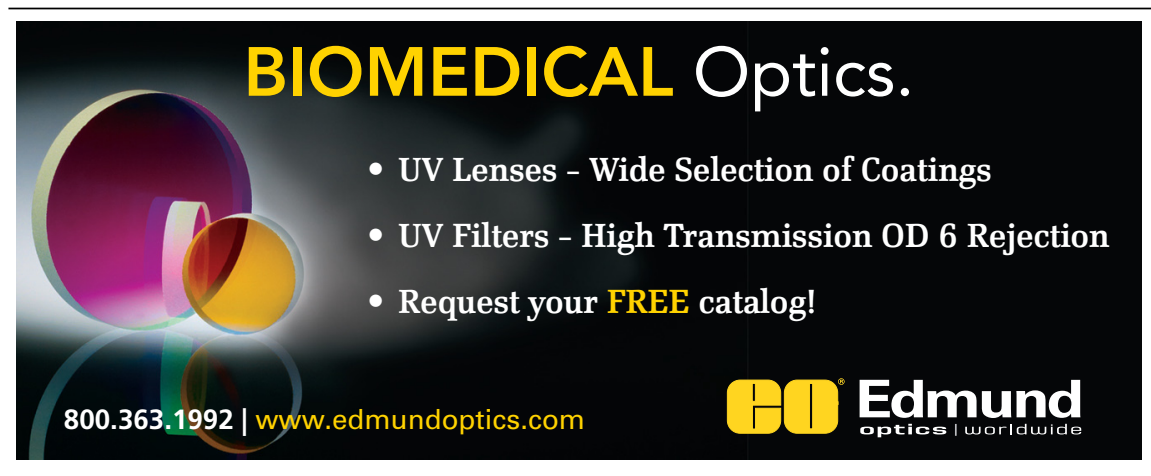

USA) containing $18 \mu \mathrm{L}$ reaction mixture. A C. parvum genomic DNA positive control (Figure 2, plot 1), the plasmid IPC (Figure 2, plot 2), a mixture of the C.parvum positive control and the IPC (Figure 2, plot 3), and a no-template control (NTC) consisting of nuclease-free water (Cat. no. AM9932; Ambion, Foster City, CA, USA) instead of the DNA (Figure 2, plot 4) were included in each experiment. The protocol took $\sim 30$ min to complete with the following PCR conditions: hot-start denaturation step at $95^{\circ} \mathrm{C}$ for $3 \mathrm{~min}$, followed by 45 cycles with a $95^{\circ} \mathrm{C}$ denaturation for $5 \mathrm{~s}, 60^{\circ} \mathrm{C}$ annealing for $30 \mathrm{~s}$ with single fluorescence acquisition (with a temperature ramp rate of $20^{\circ} \mathrm{C} / \mathrm{s}$ ). A positive result was recorded for FAM in channel F1 $(530 \mathrm{~nm})$ and for Cy5.5 in channel F3 (705 nm). Amplification reactions were carried out in triplicate. Amplicons (159 bp) were visualized by agarose gel electrophoresis and Glow-Green nucleic acid staining (eEnzyme, Gaithersburg, MD, USA) to confirm the specificity of the PCR product.

\section{Results and discussion}

Real-time PCR fluorescence signals reflecting amplification of the $C$. parvum genomic DNA was detected in the F1 channel (Figure 2A) due to FAM labeling of the TaqMan probe. Fluorescence signal reflecting amplification of the IPC was detected in the F3 channel (Figure 2B) due to FAM/Cy5.5 labeling of the IPC TaqMan probe. This probe produced an increase in Cy5.5 in the F3 channel (Cy5.5), but no increase in FAM in the F1 channel (FAM) when BHQ3 is separated from the FAM/Cy5.5 due to nuclease activity, 
because energy was transferred from FAM to Cy5.5. Similarly, in the duplex reaction, the $C$. parvum genomic DNA amplification was detected in the F1 channel, while the IPC amplification was simultaneously detected in the F3 channel. FAM has an excitation at $495 \mathrm{~nm}$ and an emission at $520 \mathrm{~nm}$. Cy5.5 has a 690-nm excitation and a 705-nm emission. With such close proximity between Cy5.5 and FAM on the triple-labeled IPC probe, the Cy5.5 fluorophore emits fluorescence due to the complete transfer of energy from the FAM fluorophore, resulting in no fluorescence emission of FAM in the F1 channel (Figure 2A). Negative control reactions did not show an increase in fluorescence due to FAM or Cy5.5. These results also demonstrate that a single blue (470 $\mathrm{nm}$ ) LED source is sufficient to monitor dual fluorescence emissions through F1 $(530 \mathrm{~nm})$ and F3 (705 nm) channels without any spectral overlap in the respective monitoring channels for FAM and Cy5.5. The FRETTaqMan technique was also tested using a panel of 12 DNA extracts from stool specimens (8 from specimens previously determined to be positive for Cryptosporidium spp. and four specimens that were negative for Cryptosporidium spp.). The $18 S$ rRNA assay was able to detect all eight Cryptosporidium spp. The IPC TaqMan assay was found to amplify only the IPC plasmid DNA. The IPC assay resulted in Ct values of 32-37, which indicated up to 5 Ct values of PCR inhibition for some stool specimens ( 32 was the expected Ct value for no-inhibition reactions).

The present technique differs from traditionally used FRET in that one fluorescently labeled oligonucleotide probe is used (FRET-TaqMan probe) instead of two probes (FRET probes) in which one probe contains the donor fluorophore and the other probe contains the acceptor fluorophore $(13,14)$. The FRET-TaqMan probe is labeled at the $5^{\prime}$ end with two fluorophores of donor (FAM) and acceptor (Cy5.5) fluorophores and at the $3^{\prime}$ end with a BHQ-3 quencher like a TaqMan probe. The unique probe-binding region is selected to differentiate from the target DNA so as to monitor two different fluorescence measurements in two different detection channels $(530$ $\mathrm{nm}$ in F1 and $705 \mathrm{~nm}$ in F3) from the same amplification reaction without the need to include color compensation software. The target DNA and IPC had the same primer binding sites but a different probe sequence. This ensures that the use of the same primers for both DNA targets to minimize primer-dimer formation or interaction between primer sets. When FAM/Cy5.5 fluorophores are placed closely at the $5^{\prime}$ end, resonance energy transfer takes place with an increase in fluorescence emission of the Cy5.5 fluorophore. However, use of BHQ3, a non-fluorescence acceptor for Cy5.5, decreases potential background fluorescence, which results in a higher signal-tonoise ratio. It was found that addition of 100 copies of the IPC to a reaction containing $\sim 100$ copies of genomic C. parvum DNA resulted in similar $\mathrm{Ct}$ values $(\mathrm{Ct}=32)$ when the Cryptosporidium TaqMan assay was performed as a singleplex assay and as a duplex assay with the IPC TaqMan assay. When applied to a panel of Cryptosporidiumpositive DNA specimens, the IPC indicated that some specimens did not contain PCR inhibitors (based on expected Ct values of 32), but other specimens were associated with inhibition of up to $5 \mathrm{Ct}$ values.

The newly developed internal PCR positive control proved to be sensitive and specific for simultaneous detection of two targets in the same reaction due to the labeling with the FAM/Cy5.5-BHQ3 and FAM/BHQ1 for the respective IPC and $18 \mathrm{~S}$ rRNA target gene in a dualcolor multiplex PCR for monitoring in F1 and F3 fluorescence channels of the R.A.P.I.D instrument. The use of the 5'FAM-Cy5.5/3'BHQ-3-labeled probe as the second reporter is advantageous because the emission maxima of the two probes were well-spaced from each other. The FRET-TaqMan triple-labeled probe design could have applications in developing an inexpensive, disposable "lab-ona-chip" device with optics consisting of one blue LED excitation and two emission sources of $530 \mathrm{~nm}$ and $705 \mathrm{~nm}$, with appropriate optical filters to detect FAM in one channel and Cy5.5 in the other. This combination would not require two high-powered fluorescence source and detection systems.

\section{Acknowledgments}

The authors acknowledge the assistance of Mike Powers at the DNA Sequencing and Genomic Core Facility (University of Utah, Salt Lake City, UT, USA) and Rachelle Muller at Idaho Technology, Inc. for discussion on the development of the internal positive control for the R.A.P.I.D cycler. This publication was supported in part by funds made available through the Centers for Disease Control and Prevention, Coordinating Office for Terrorism Preparedness and Emergency Response. Use of trade names and commercial sources is for identification only and does not imply endorsement by the Centers for Disease Control and Prevention or the U.S. Department of Health and Human Services.

\section{References}

1. Hartman, L.J., S.R. Coyne, and D.A. Norwood. 2005. Development of a novel internal positive control for Taqman based assays. Mol. Cell. Probes 19:51-59.

2. Hoorfar, J., B. Malorny, A. Abdulmawjood, N. Cook, M. Wagner, and P. Fach. 2004. Practical considerations in design of internal amplification controls for diagnostic PCR assays. J. Clin. Microbiol. 42:1863-1868.

3. Betsou, F., K. Beaumont, J.M. Sueur, and J. Orfila. 2003. Construction and evaluation of internal control DNA for PCR amplification of Chlamydia trachomatis DNA from urine samples. J. Clin. Microbiol. 41:1274-1276.

4. Burggraf, S. and B. Olgemoller. 2004. Simple technique for internal control of real-time amplification assays. Clin. Chem. 50:819-825.

5. Drosten, C., M. Weber, E. Seifried, and W.K. Roth. 2000. Evaluation of a new PCR assay with competitive internal control sequence for blood donor screening. Transfusion 40:718-724.

6. Gruber, F., F.G. Falkner, F. Dorner, and T. Hammerle. 2001. Quantitation of viral DNA by real-time PCR applying duplex amplification, internal standardization, and two-color fluorescence detection. Appl. Environ. Microbiol. 67:2837-2839.

7. Rosenstraus, M., Z. Wang, S.Y. Chang, D. DeBonville, and J.P.Spadoro. 1998. An internal control for routine diagnostic PCR: design, properties, and effect on clinical performance. J. Clin. Microbiol. 36:191-197.

8. Stocher, M., V. Leb, and J. Berg. 2003. A convenient approach to the generation of multiple internal control DNA for a panel of real-time PCR assays. J. Virol. Methods 108:1-8.

9. Zimmermann, K. and J.W. Mannhalter. 1996. Technical aspects of quantitative competitive PCR. BioTechniques 21:268-279.

10. Jothikumar, N., A.J. da Silva, I. Moura, Y. Qvarnstrom, and V.R. Hill. 2008. Detection and differentiation of Cryptosporidium hominis and Cryptosporidium parvum by dual TaqMan assays. J. Med. Microbiol. 57:1099-1105.

11. Altschul, S.F., W. Gish, W. Miller, E.W. Myers, and D.J. Lipman. 1990. Basic local alignment search tool. J. Mol. Biol. 215:403-410.

12. Hill, V.R., A.M. Kahler, N. Jothikumar, T.B. Johnson, D. Hahn, and T.L. Cromeans. 2007. Multistate evaluation of an ultrafiltrationbased procedure for simultaneous recovery of enteric microbes in 100-liter tap water samples. Appl. Environ. Microbiol. 73:4218-4225.

13. Bernard, P.S., M.J. Lay, and C.T Wittwer. 1998. Integrated amplification and detection of the $\mathrm{C677T}$ point mutation in the methylenetetrahydrofolate reductase gene by fluorescence resonance energy transfer and probe melting curves. Anal. Biochem. 255:101-107.

14. Wittwer, C.T., M.G. Herrmann, A.A.Moss, and R.P. Rasmussen. 1997. Continuous fluorescence monitoring of rapid cycle DNA amplification. BioTechniques 22:130-138.

Received 20 November 2009; accepted 9 February 2009.

Address correspondence to Jothikumar Narayanan, Centers for Disease Control and Prevention, National Center for Zoonotic, Vector-borne, and Enteric Diseases, Division of Parasitic Diseases, Atlanta, GA, 30341, USA. email: jin2@cdc.gov 